\title{
BMJ Open Recruitment, adherence, and retention of endometrial cancer survivors in a behavioural lifestyle programme: the Diet and Exercise in Uterine Cancer Survivors (DEUS) parallel randomised pilot trial
}

\author{
Dimitrios A Koutoukidis, ${ }^{1,2}$ Rebecca J Beeken, ${ }^{2,3}$ Ranjit Manchanda, ${ }^{1,4,5}$ \\ Moscho Michalopoulou, ${ }^{1}$ Matthew Burnell, ${ }^{1}$ M Tish Knobf, ${ }^{1,6}$ Anne Lanceley ${ }^{1}$
}

To cite: Koutoukidis DA, Beeken RJ, Manchanda R, et al. Recruitment, adherence, and retention of endometrial cancer survivors in a behavioural lifestyle programme: the Diet and Exercise in Uterine Cancer Survivors (DEUS) parallel randomised pilot trial. BMJ Open 2017;7:e018015. doi:10.1136/ bmjopen-2017-018015

- Prepublication history and additional material for this paper are available online. To view please visit the journal (http:// dx.doi.org/10.1136/bmjopen2017-018015).

Received 31 May 2017 Revised 12 July 2017 Accepted 3 August 2017

CrossMark

For numbered affiliations see end of article.

Correspondence to

Dr Anne Lanceley;

a.lanceley@ucl.ac.uk

\section{ABSTRACT}

Objective Healthy eating and physical activity may help endometrial cancer survivors (ECS) improve their quality of life. However, most ECS do not meet the relevant guidelines. This pilot trial aimed to test the study feasibility procedures for a definitive trial of a behavioural lifestyle programme.

Design and setting This 24-week parallel two-arm randomised pilot trial took place in two hospitals in London, UK (April 2015-June 2016).

Participants Sixty disease-free ECS within 3 years of diagnosis.

Interventions Participants were randomised using minimisation to receive the intervention or care as usual. The 'Shape-Up following cancer treatment' programme used self-monitoring, goal-setting, self-incentives, problem-solving and group social support for 12 hours over 8 weeks to help survivors improve their eating and physical activity.

Outcome measures The main outcome measures were recruitment, adherence, and retention rates. Further outcomes included barriers to participation and feedback on programme satisfaction.

Results Of the 296 potentially eligible ECS, $20 \%$ ( $n=60$ ) were randomly allocated to the active intervention $(n=29)$ or control group $(n=31)$. Three participants in each arm were deemed ineligible after randomisation and excluded from analysis. Twenty participants $(77 \%$; $95 \% \mathrm{Cl} 61 \%$ to $93 \%$ ) adhered to the intervention and provided generally favourable feedback. At 24 weeks, 25/26 (96\%; 95\% Cl $89 \%$ to $100 \%$ ) intervention and $24 / 28$ (86\%; $95 \% \mathrm{Cl} 73 \%$ to $99 \%$ ) control participants completed their assessment. No intervention-related adverse events were reported. Among eligible survivors who declined study participation $(\mathrm{n}=83)$, inconvenience $(78 \% ; 95 \% \mathrm{Cl} 69 \%$ to $87 \%)$ was the most common barrier.

Conclusions The trial was feasible to deliver based on the a priori feasibility criteria. Enhancing recruitment and adherence in a definitive trial will require designs that promote convenience and consider ECS-reported barriers. Trial registration number NCT02433080; Pre-results.
Strengths and limitations of this study

- This trial tested the feasibility of a standardised theory-based behavioural lifestyle programme for endometrial cancer survivors using a robust randomised parallel design.

- Barriers to participation were systematically assessed.

- The study aimed to minimise these barriers by recruiting survivors within the 'teachable moment' period and capitalising on the endorsement of the study from their clinicians.

- The small sample size and recruitment from London-based hospitals limit the generalisability of the outcomes.

Trial funding University College London, St. Bartholomew's Hospital Nurses League, and NIHR University College London Hospitals Biomedical Research Centre.

\section{INTRODUCTION}

Endometrial cancer is the most common gynaecological cancer with about 455000 incident cases worldwide in 2015. It affects mostly women in developed countries ${ }^{1}$ and about $75 \%$ women will live for more than 10 years after diagnosis. ${ }^{2}$ They are the cancer group with the highest comorbidity burden among survivors ${ }^{3}$ and are most likely to die from cardiovascular disease. ${ }^{4}$ Furthermore, the prevalence of obesity and suboptimal lifestyle behaviours is high, both of which are associated with lower health-related quality of life. ${ }^{5}$ Although most survivors do not spontaneously adopt health-protective behaviours ${ }^{6}$ postdiagnosis, they do report trying to make 
lifestyle changes. However, they experience cancer-specific barriers, such as fatigue and bowel issues, and feel there is a lack of guidance. ${ }^{7}$

Behavioural lifestyle interventions improve patient-reported outcomes, such as health-related quality of life, in other cancer survivor groups. ${ }^{8-10}$ Randomised controlled trials (RCTs) in endometrial cancer survivors (ECS) have also shown that health behaviour change is feasible for these patients. ${ }^{11} 12$ However, the programmes tested to date have been resource-intensive rendering their widespread dissemination challenging. There is, therefore, a need for effective lifestyle behaviour change interventions that can be adopted within the cancer care pathway. We have adapted an existing evidence-based lifestyle intervention, ${ }^{13}$ which is already running within the healthcare system, to try and facilitate this process. ${ }^{14}$ The intervention was adapted to the particular needs and preferences of ECS, with patient input and using the intervention mapping approach. A definitive RCT will indicate whether this intervention is effective in promoting longterm behaviour change and improving survivors' quality of life. This pilot study was conducted to test the feasibility of the planned RCT's procedures.

The primary objective of the pilot trial was therefore to calculate recruitment, adherence, and retention rates. Secondary outcomes included willingness of clinical staff to recruit participants, potential adverse events, barriers to participation, reasons for attrition, and participants' study experience. ${ }^{15}$

\section{METHODS}

\section{Study design and participants}

The trial protocol has been published. ${ }^{15}$ The DEUS (Diet and Exercise in Uterine Cancer Survivors) pilot trial was an 8-week, two-arm, parallel, controlled pilot trial with 1:1 randomisation comparing the use of the 'Shape-Up following cancer treatment' programme to care as usual.

Women aged $\geq 18$ years who had been diagnosed with endometrial cancer (ICD C54.1) within the previous 36 months were eligible to take part in the study. Women were excluded if (1) they were diagnosed with stage IVB cancer; (2) they were on active anticancer and/or palliative treatment; (3) they had a second primary cancer; (4) they lacked mental capacity to decide to take part in the study and to participate in it; (5) they had severe depression; (6) they were unavailable for longitudinal follow-up assessments; (7) they had participated in a professionally delivered weight loss or exercise programme during the previous 6 months; (8) their performance score was $3-4^{16}(9)$ or they were unable to understand spoken and written English.

At the fifth week of recruitment, the inclusion criterion 'women willing to attend all sessions' was removed given the subjective nature of its interpretation and the exclusion criterion 'women with secondary cancer' was added to ensure homogeneity.

\section{Recruitment}

Potential participants were recruited from the gynaecology outpatient clinics at University College London Hospitals (UCLH) and Barts Health. Interested and potentially eligible participants were introduced to the study by clinicians and researchers attending the clinics as previously described. ${ }^{15}$

The clinicians at UCLH also identified potential participants that had been treated there but followed up at local sites. Following general practitioner's verification that the patients were alive, invitation letters signed by the consultant were sent to these women together with the participant information sheet, an opt-in form, a barriers to participation survey and a business reply envelope.

\section{Randomisation and blinding}

Consented participants were randomised with a 1:1 allocation to receive either the intervention or usual care through minimisation using age and body mass index (BMI) as stratified variables. The process has been previously described in detail. ${ }^{15}$ The researcher assessing the 8-week outcomes (MM) was blinded to intervention allocation and participants were requested prior to the assessment not to disclose their allocation.

\section{Shape-Up following cancer treatment intervention}

In addition to usual care, intervention-arm participants received the 'Shape-Up following cancer treatment' manual and were assigned to groups of three to eight, although the initial plan was that they would be assigned in groups of eight. The allocation to groups was on a firstcome, first-served basis to avoid delays in delivering the intervention to randomised participants and aimed to match participant preferences for dates and times of the group meetings. The five groups met weekly for 8 weeks at UCLH. Each session lasted approximately $90 \mathrm{~min}$. The theory-based intervention has been previously described. ${ }^{15}$ In brief, it included advice on healthy eating, physical activity, management of triggers of unhealthy behaviours and behavioural relapse prevention. A dietitian (DAK) trained on the programme facilitated the group sessions following a standardised and scripted protocol. An extra trained provider (psychologist or dietitian) attended the meetings of the four groups to aid with facilitation but did not participate in the discussion. DAK was the only facilitator in the last group because of last minute cancellations. The participants in the fourth and final round of randomisation were split into two small intervention groups for convenience purposes. A participant from the control group who had completed the study was invited to participate in the last group (final $n=4$ in group 5) to enhance the group experience but was not included in the analysis. There were no other modifications. The completed Consolidated Standards of Reporting Trials (CONSORT) ${ }^{17}$ and Template for Intervention Description and Replication (TIDieR) checklists ${ }^{18}$ are available in online supplementary appendices S1 and S2, respectively. 


\section{Care as usual}

Participants in the control arm were offered usual care. After the final follow-up, they received a booklet with healthy lifestyle advice for cancer survivors. ${ }^{19}$

\section{OUTCOMES}

Recruitment rates were calculated separately for each strategy and site. We adapted an existing framework of hierarchical recruitment barriers (availability by disease characteristics, eligibility, physician triage, trial discussion, interest, consent, and enrolment) ${ }^{20}$ to describe the recruitment process. In contrast with the original framework, the category 'interest' preceded that of 'trial discussed' to fit the current recruitment process. Participants who were introduced to the study and decided not to enrol completed a 25-item investigator-designed survey ${ }^{21}$ about barriers to participation. Eight UCLH clinicians were interviewed about their views on study recruitment using a semistructured protocol over phone or face-to-face (online supplementary appendix S3).

Participants attended a 90-min baseline site visit with a trained researcher (DAK) to complete their measurements and questionnaires. The visit was repeated at 8 and 24 weeks with MM and DAK, respectively. All intervention sessions were audiotaped. RJB attended one intervention session and one study assessment and scored them against a predefined checklist. Engaged intervention-arm participants completed and posted an 18-item programme evaluation questionnaire. ${ }^{22}$ Only two follow-up qualitative interviews with intervention participants were performed at study completion, as the data from the open-ended feedback questionnaire were deemed sufficient.

\section{Statistical and qualitative analysis}

Despite the pilot nature of the study, a sample size of 32 participants per arm was estimated for examining recruitment, adherence, and retention rates. The study would be deemed feasible if the lower 95\% CIs for recruitment, adherence and retention rates were at least $15 \%, 60 \%$, and $60 \%$, respectively. ${ }^{15}$

Primary outcomes are reported in proportions with 95\% CIs. Descriptive statistics are reported for continuous variables. Categorical variables are summarised using frequencies and percentages. The interviews with clinicians lasted $10 \mathrm{~min}$ on average, were digitally recorded, transcribed verbatim by a professional company and checked for accuracy. Given the structured interview and short replies, data were analysed with content analysis using NVivo V.10 (QSR International, 2014) software. The open-ended questions were analysed using manifest content analysis ${ }^{23}$ in Microsoft Office Excel V.2011. This process involved determining the frequency of words and content in the text.
RESULTS

\section{Recruitment}

Recruitment took place over a period of 27 and 18 weeks (April 2015-December 2015) at UCLH and Barts Health, respectively (figure $\mathrm{S} 1$ of online supplementary appendix S3). The difference in recruitment period between sites was primarily explained by substantial delay of NHS Research and Development management approval at Barts Health. Among the first 64 eligible participants approached, 20 consented to participate, leading to rejection of the null hypothesis that recruitment would be $\leq 15 \%$. Therefore, recruitment continued for enrolling the projected sample of 64 participants but stopped after enrolling 60 participants due to resource constraints. Out of 296 potentially eligible participants, 20.3\% (95\% CI $15.7 \%$ to $24.9 \%$ ) enrolled in the study. Among screened participants, rates of consent were similar for the face-toface recruitment at the two recruitment sites but lower for the mail-out (tables S1 and S2 of online supplementary appendix S3).

Reasons for non-participation were documented for $36.7 \%(n=83)$ of those who were potentially eligible but did not consent and $90.2 \%$ of those that were approached. Inconvenience to everyday life $(78 \%)$ and transport to trial site $(63 \%)$ were the main barriers to participation, with further barriers detailed in table 1 and less frequent barriers in table S3 of online supplementary appendix 3. The CONSORT flow diagram shows the progress through the trial stages (figure 1).

\section{Clinicians' views on recruitment}

Clinicians were supportive of the study and did not have particular concerns about introducing the study to patients. They felt the study might be beneficial to patients, but they believed travelling and commitment would be the main barriers for recruitment.

They deemed the recruitment strategy highly effective, with potentially eligible patients being flagged prior to the clinic, researchers being present and reminding them about approaching patients, and through the existence of a separate space for study recruitment in the clinic. These strategies minimised additional clinician workload.

Clinicians did not anticipate adverse events from the intervention or changes in their relationship with the patients. The framing and content of such an intervention was also highlighted as a potential barrier to recruitment. In particular, approaching patients in a non-discriminatory way was deemed to enhance recruitment. Furthermore, framing of its content as a lifestyle programme was thought to be superior to a weight loss programme, strict diet regime or educational programme.

\section{Sample characteristics}

Participant characteristics at baseline are shown in table 2. Women were on average $( \pm \mathrm{SD}) 62.1 \pm 8.3$ years old, white $(67 \%)$, married $(53 \%), 1.2 \pm 1.0$ years from diagnosis, with a BMI of $28.0 \pm 6.3 \mathrm{~kg} / \mathrm{m}^{2}$. They were diagnosed mostly with stage IA (49\%), type $1(82 \%)$ endometrial cancer. 
Table 1 Percentage of each barrier to participation with SE among eligible survivors who declined participation $(n=83)$

\begin{tabular}{ll}
\hline Barrier to participation & $\%$ (SE) \\
\hline Inconvenient to everyday life & $78.3(4.5)$ \\
\hline Transport or distance to trial site & $62.7(5.3)$ \\
\hline Feelings of uncertainty & $15.7(4.0)$ \\
\hline Lack of family support & $15.7(4.0)$ \\
\hline The design of the study is too difficult to & $15.7(4.0)$ \\
\hline understand or too binding & $15.7(4.0)$ \\
\hline Trial or treatment has no benefits & $14.5(3.9)$ \\
\hline Other: feeling physically unwell & $13.3(3.7)$ \\
\hline Preference for other treatment (eg, Weight & $12.1(3.6)$ \\
\hline Watchers) & $12.1(3.6)$ \\
\hline Increased anxiety & $12.1(3.6)$ \\
\hline Quality of life might be reduced & $9.6(3.2)$ \\
\hline Trial or treatment does not offer best option & $9.6(3.2)$ \\
\hline Trial setting & \\
\hline Uncomfortable with experimentation & $7.2(2.8)$ \\
\hline Do not want to lose control of decision- & $7.2(2.8)$ \\
\hline making & $6.0(2.6)$ \\
\hline General unease with research process & $4.8(2.4)$ \\
\hline Other: does not like to discuss in groups & $4.8(2.4)$ \\
\hline Belief that doctor should make decisions & 4.4 (2.4) \\
\hline Dislike idea of randomisation & \\
\hline Trials not appropriate for serious disease & \\
\hline
\end{tabular}

\section{Adherence}

Out of 26 participants in the intervention arm, 21 (81\%; $95 \%$ CI $66 \%$ to $96 \%)$ engaged and 20 (77\%; 95\% CI $61 \%$ to $93 \%$ ) adhered to the intervention, based on our predetermined criteria. ${ }^{15}$ The lower CI was above $60 \%$ indicating feasibility.

The percentage of participants who attended zero, five, six, seven, and eight (all) sessions was 15\%, $8 \%$, $12 \%, 35 \%$, and $15 \%$, respectively. The mean overall attendance of sessions was $63 \%$ (95\% CI $49 \%$ to $77 \%$ ). The mean attendance rates of those who engaged and those who adhered were $79 \%(95 \%$ CI $70 \%$ to $88 \%)$ and $82 \%$ (95\% CI $74 \%$ to $89 \%$ ), respectively. Reasons for not commencing the intervention (ie, attendance of zero sessions) included inconvenience to everyday life $(n=1)$, family commitments $(n=1)$, lack of perceived benefit $(n=1)$ or travel burden $(n=1)$. The remaining two dropouts occurred in the first two sessions due to programme content and travelling abroad. Absence from the group sessions among adhered participants were mostly of practical nature, with details available in online supplementary appendix S3.

\section{Programme satisfaction}

Eighteen participants randomised to the intervention group who adhered provided feedback for the programme. They scored the programme highly with $44 \%$ and $39 \%$ reporting that it met or exceeded their expectations, respectively. All aspects of the programme were scored highly (table 3 ). Additionally, most participants ranked self-monitoring, setting specific, measurable, achievable, relevant, and time-specific goals, and social support as either very or somewhat helpful in making dietary and physical activity changes (table 4). In contrast, the responses for self-incentives were mixed with $28 \%$ of participants rating this technique as unhelpful.

A range of topics were regarded as most useful (figure S2 of online supplementary appendix S3). Among them, most participants agreed that the sections about keeping an eye on portion sizes, food labelling and internal triggers were the most useful. Others mentioned self-incentives, internal and external triggers and getting a healthier balance of foods to be the least useful topics (figure S3 of online supplementary appendix 3). For example, one participant mentioned:

I also did not understand the concept of the rewardsbetter health should be its own reward (Participant in group 4).

Suggestions for additions to the programme were primarily focused on physical activity, such as provision of relevant digital video discs, physical activity during the programme sessions and diaries to report physical activity and sedentary behaviour in more detail. Most participants did not consider that topics should be eliminated from the programme. Similar feedback was provided for the booklet; most participants did not suggest changes while a few suggested design changes. Further suggestions included the addition of follow-up support and a preference for a larger group (mentioned by participants in smaller groups) to boost the peer-education component.

Peer support of the group, both the focus of the programme and their own interest in health promotion, the feeling of giving back to the care system, the facilitators and the doctor's referral to the programme facilitated study participation. In contrast, most did not report factors discouraging them to participate, but some mentioned inconvenience to everyday life, self-monitoring, and identification as a cancer survivor.

Regarding the trial procedures, two participants mentioned their difficulty recalling and quantifying their diet and physical activity. Excellent fidelity to the protocol for both the group sessions $(85 \%)$ and the assessments $(100 \%)$ was demonstrated in the study auditing.

\section{Retention}

Retention rate was $92 \%$ (95\% CI $85 \%$ to $100 \%)$, with 24/28 (86\%; 95\% CI $73 \%$ to $99 \%)$ and $25 / 26(96 \%$; $95 \%$ CI $89 \%$ to $100 \%$ ) eligible participants in the control and intervention arm completing all assessments, respectively ( $\mathrm{p}=0.61$ for difference between proportions). This 


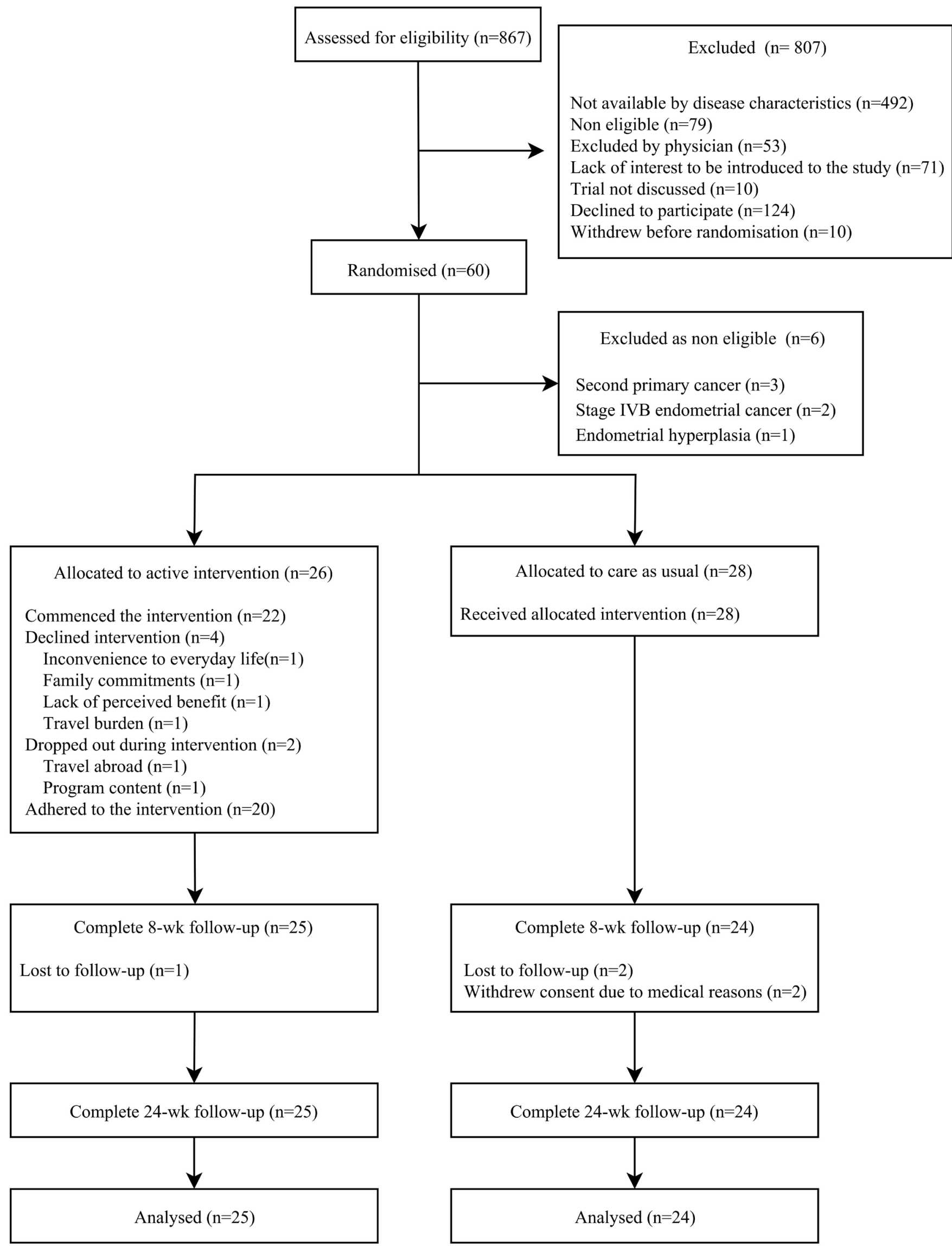

Figure 1 Consolidated Standards of Reporting Trials diagram of the trial with framework on barriers to participation in the exclusion box. wk, week. 
Open Access

Table 2 DEUS pilot trial baseline participant characteristics

\section{Characteristic}

Age, mean (SD)

Race

\begin{tabular}{|c|c|c|c|}
\hline White & $17(68)$ & $16(67)$ & $33(67)$ \\
\hline Asian & $4(16)$ & $5(21)$ & $9(18)$ \\
\hline Other & $4(16)$ & $3(12)$ & $7(14)$ \\
\hline \multicolumn{4}{|l|}{ Living arrangement } \\
\hline Own outright/mortgage & $17(68)$ & $15(63)$ & $32(65)$ \\
\hline Rent & $8(30)$ & $9(38)$ & $17(35)$ \\
\hline \multicolumn{4}{|l|}{ Marital status } \\
\hline Married/living with partner/civil partnership & $12(48)$ & $15(63)$ & $27(55)$ \\
\hline Separated/divorced & $7(28)$ & $3(12)$ & $10(20)$ \\
\hline Widowed/single & $6(24)$ & $6(25)$ & $12(24)$ \\
\hline \multicolumn{4}{|l|}{ Education } \\
\hline $\begin{array}{l}\text { Degree/higher degree/higher education below } \\
\text { degree level }\end{array}$ & $11(44)$ & $12(50)$ & $23(47)$ \\
\hline Secondary education & $11(44)$ & $10(42)$ & $21(42)$ \\
\hline No formal qualifications & $3(12)$ & $2(8)$ & $5(10)$ \\
\hline \multicolumn{4}{|l|}{ Employment } \\
\hline Full time/self-employed & $9(36)$ & $11(46)$ & $20(41)$ \\
\hline Part time/other & $6(24)$ & $2(8)$ & $8(16)$ \\
\hline Retired & $10(40)$ & $11(46)$ & $21(43)$ \\
\hline \multicolumn{4}{|l|}{ Smoking } \\
\hline Current & $2(8)$ & $2(8)$ & $4(8)$ \\
\hline Former & $4(16)$ & $5(21)$ & $9(18)$ \\
\hline \multicolumn{4}{|l|}{ IMD (quintile) } \\
\hline 1 (most deprived) & $5(20)$ & $4(17)$ & $9(18)$ \\
\hline 2 & $9(36)$ & $6(25)$ & $15(31)$ \\
\hline 3 & $4(16)$ & $7(29)$ & $11(22)$ \\
\hline 4 & $3(12)$ & $3(13)$ & $6(12)$ \\
\hline 5 (least deprived) & $4(16)$ & $4(17)$ & $8(16)$ \\
\hline Time since diagnosis in months, mean (SD) & $19.2(11.2)$ & $21.4(11.3)$ & $20.3(11.2)$ \\
\hline $\begin{array}{l}\text { Time since completion of primary treatment in } \\
\text { months, mean (SD) }\end{array}$ & $17.1(11.2)$ & $18.5(11.7)$ & 17.8 (11.3) \\
\hline Surgery & $25(100)$ & $24(100)$ & $49(100)$ \\
\hline Chemotherapy treatment & $3(12)$ & $5(21)$ & $8(16)$ \\
\hline External beam radiotherapy & $6(24)$ & $12(50)$ & $18(37)$ \\
\hline Brachytherapy & $11(44)$ & $13(54)$ & $24(49)$ \\
\hline \multicolumn{4}{|l|}{ Cancer stage } \\
\hline IA & $11(44)$ & $13(54)$ & 24 (49) \\
\hline IB & $11(44)$ & $6(25)$ & $17(35)$ \\
\hline II & $2(8)$ & $3(13)$ & $5(10)$ \\
\hline IIIA & $1(4)$ & $2(8)$ & $3(6)$ \\
\hline \multicolumn{4}{|l|}{ Cancer grade } \\
\hline 1 & $6(24)$ & 7 (29) & $13(27)$ \\
\hline 2 & $13(52)$ & $9(38)$ & $22(45)$ \\
\hline
\end{tabular}

Continued 


\begin{tabular}{|c|c|c|c|}
\hline Characteristic & Shape-Up $(n=25)$ & Care as usual $(n=24)$ & Total \\
\hline 3 & $6(24)$ & $8(33)$ & $14(29)$ \\
\hline \multicolumn{4}{|l|}{ Histology } \\
\hline Endometrioid adenocarcinoma & $21(84)$ & $19(79)$ & $40(82)$ \\
\hline Serous carcinoma & $1(4)$ & $3(13)$ & $4(8)$ \\
\hline Mixed carcinoma & $1(4)$ & $0(0)$ & $1(2)$ \\
\hline Serous surface papillary carcinoma & $0(0)$ & $1(4)$ & $1(2)$ \\
\hline Carcinosarcoma & $2(8)$ & $0(0)$ & $2(4)$ \\
\hline Adenosquamous carcinoma & $0(0)$ & $1(4)$ & $1(2)$ \\
\hline \multicolumn{4}{|l|}{ Histological type } \\
\hline Type I & $21(84)$ & $19(79)$ & $40(82)$ \\
\hline Type II & $4(16)$ & $5(21)$ & $9(18)$ \\
\hline \multicolumn{4}{|l|}{ Charlson Comorbidity Index } \\
\hline 2 & $18(75)$ & $21(84)$ & $39(80)$ \\
\hline 3 & $6(25)$ & $4(16)$ & $10(20)$ \\
\hline \multicolumn{4}{|l|}{ WHO performance status } \\
\hline 0 & $20(83)$ & $20(80)$ & $40(82)$ \\
\hline 1 & $3(13)$ & $5(20)$ & $8(16)$ \\
\hline 2 & $1(4)$ & $0(0)$ & $1(2)$ \\
\hline \multicolumn{4}{|l|}{ Selected comorbidities } \\
\hline Diabetes & $3(12)$ & $4(17)$ & 7 (14) \\
\hline Hypertension & $6(24)$ & 7 (29) & $13(27)$ \\
\hline Dyslipidaemia & $3(12)$ & $3(13)$ & $6(12)$ \\
\hline Asthma & $1(4)$ & $2(8)$ & $3(6)$ \\
\hline Osteoporosis & $2(8)$ & $4(17)$ & $6(12)$ \\
\hline Weight, mean kg (SD) & $69.8(14.8)$ & $71.9(15.2)$ & $70.9(14.9)$ \\
\hline $\mathrm{BMI}$, mean kg/m² (SD) & $27.3(6.5)$ & $28.8(6.1)$ & $28.0(6.3)$ \\
\hline BMI, median kg/m² (IQR) & $26.2(24.3)$ & $26.9(8.6)$ & $26.8(61.4)$ \\
\hline \% Fat, mean (SD) & $35.3(7.7)$ & $36.9(6.3)$ & $36.1(7.0)$ \\
\hline
\end{tabular}

Percentages might not add to 100 due to rounding.

Data are presented as n (\%) unless otherwise specified.

Body composition data for usual care $\mathrm{n}=23$.

BMI, body mass index; DEUS, Diet and Exercise in Uterine Cancer Survivors; IMD, index of multiple deprivation.

indicated an absence of attrition bias and the rejection of the null hypothesis that retention rate would be less than $60 \%$.

\section{Adverse events and control arm contamination}

No intervention-related adverse events or unintended consequences were reported. Adverse events unrelated to the intervention and reasons for control arm contamination are detailed in the online supplementary appendix S3.

\section{DISCUSSION}

This is the first pilot study of a health behaviour change intervention in ECS in the UK to demonstrate feasibility in terms of recruitment, adherence, and retention. The collaboration of the clinical and research team led to an efficient recruitment process. Participants rated the programme highly and provided rich feedback for refinement. Consistent with the literature ${ }^{24}$ and the qualitative findings, ${ }^{7}$ the DEUS pilot study aimed to minimise accrual barriers by enrolling survivors within the 'teachable moment' period, capitalising on the endorsement of the study from survivors' clinicians, using a strong behaviour theory-based design and ensuring standardised delivery of the intervention. These study strengths were also reflected in the reported factors associated with programme involvement. Furthermore, the frameworks for reporting barriers to participation ${ }^{20}{ }^{21}$ provided a comprehensive understanding of these barriers and can be a valuable resource to understand barriers in for future 
Table 3 Percentage programme satisfaction $(n=18)$

\begin{tabular}{|c|c|c|c|}
\hline $\begin{array}{l}\text { How much did you like } \\
\text { the... }\end{array}$ & $\begin{array}{l}\text { Dislike } \\
(\%)\end{array}$ & $\begin{array}{l}\text { Neither like } \\
\text { or } \\
\text { dislike (\%) }\end{array}$ & $\begin{array}{l}\text { Like } \\
(\%)\end{array}$ \\
\hline $\begin{array}{l}\text { Organisation of the } \\
\text { sessions }\end{array}$ & - & - & 100 \\
\hline Length of the programme & - & 11 & 89 \\
\hline Dates of the programme & 6 & 17 & 78 \\
\hline Length of the sessions & - & - & 100 \\
\hline Training location & - & - & 100 \\
\hline Group format & - & - & 100 \\
\hline Peer-education format & - & 6 & 94 \\
\hline Group discussion & - & - & 100 \\
\hline $\begin{array}{l}\text { Cultural sensitivity of the } \\
\text { facilitator }\end{array}$ & - & - & 100 \\
\hline $\begin{array}{l}\text { Facilitator's knowledge of } \\
\text { materials }\end{array}$ & - & 6 & 94 \\
\hline Facilitator's preparedness & - & - & 100 \\
\hline $\begin{array}{l}\text { Time used effectively by } \\
\text { facilitator }\end{array}$ & - & - & 100 \\
\hline $\begin{array}{l}\text { Attractiveness of the } \\
\text { booklet }\end{array}$ & 6 & 17 & 78 \\
\hline $\begin{array}{l}\text { Overall design of the } \\
\text { booklet }\end{array}$ & - & 17 & 84 \\
\hline Wording of the booklet & - & 22 & 77 \\
\hline Volume of the booklet & 17 & 22 & 61 \\
\hline Durability of the booklet & - & 17 & 83 \\
\hline
\end{tabular}

trials. ${ }^{25}$ Limitations of the study include the small sample size, recruitment from only two London-based sites, and generalisability of the recruited sample, as sociodemographic data from decliners were missing. The relatively low median BMI of participants compared with epidemiological studies ${ }^{26}$ indicates healthy volunteer effect biases. The wide socioeconomic and demographic differences of the population pools of the two hospitals ${ }^{27}$ and the similar recruitment rates at both sites were reassuring and suggest these factors should not impact recruitment and retention.

The focus of the study on healthy lifestyle changes rather than weight loss was postulated to increase uptake and acceptability of the programme. ${ }^{7}$ The overall recruitment estimate was similar or somewhat higher than that in other lifestyle intervention trials, although differences in recruitment strategies, eligibility criteria, cancer site, programme length and intensity do not allow for direct comparisons. The group-based, 6-month Survivors of Uterine Cancer Empowered by Exercise and Healthy Diet (SUCCEED) intervention had a $19 \%$ recruitment rate using mail-out. ${ }^{12}$ A 12-week group-based physical activity intervention recruited $20 \%$ of the eligible ECS through fliers and telephone recruitment. ${ }^{28}$ Similar to DEUS, a more intensive lifestyle intervention in UK breast cancer survivors had a mail-out rate of $17 \% .{ }^{29}$ While removing the transport and time barriers would theoretically improve recruitment rates, USA home-based lifestyle interventions recruiting cancer survivors from registries

Table 4 Helpfulness of the main behaviour change techniques for dietary and physical activity changes $(n=18)$

\begin{tabular}{|c|c|c|c|}
\hline & Unhelpful (\%) & $\begin{array}{l}\text { Neither } \\
\text { helpful or } \\
\text { unhelpful (\%) }\end{array}$ & Helpful (\%) \\
\hline $\begin{array}{l}\text { When trying to make diet changes, how helpful did you find monitoring } \\
\text { yourself using diaries? }\end{array}$ & - & - & 100 \\
\hline $\begin{array}{l}\text { When trying to make physical activity changes, how helpful did you find } \\
\text { monitoring yourself using diaries? }\end{array}$ & $6 \%$ & 6 & 88 \\
\hline $\begin{array}{l}\text { When trying to make physical activity changes, how helpful did you find } \\
\text { putting SMART goals? }\end{array}$ & - & 11 & 89 \\
\hline $\begin{array}{l}\text { When trying to make diet changes, how helpful did you find rewarding } \\
\text { yourself? }\end{array}$ & $28 \%$ & 33 & 39 \\
\hline $\begin{array}{l}\text { When trying to make physical activity changes, how helpful did you find the } \\
\text { peer-education nature of the sessions? }\end{array}$ & - & 17 & 83 \\
\hline
\end{tabular}


have shown much smaller recruitment rates $(5.7 \%)$ with women, younger, white survivors, and those closer to their cancer diagnosis more likely to enrol. ${ }^{30}$

The observed adherence was lower compared with the weight loss SUCCEED intervention $(84.1 \%)$ comprising 16 group sessions ${ }^{12}$ but similar to that of a group-based 12-week physical activity intervention. ${ }^{28}$ While this might indicate that survivors are more committed in weight loss programmes compared with healthy lifestyle programmes, the main reported reasons regarding non-attendance in the current study were around practicalities and life commitments rather than disengagement with the programme. Sending a standardised email to non-attendees about topics covered in the missed session and preparation for the next session helped maintain their engagement.

Having a specific research room and two committed researchers in clinic facilitated recruitment. Screening participants using electronic forms and implementing further prerandomisation eligibility checks from medical notes could minimise randomisation of ineligible participants. The recruitment rate, while similar between the two sites, was lower in the clinician-endorsed mail-out, indicating the higher effectiveness of the first approach that needs to be balanced with its higher resource requirements in larger trials. Practical reasons rendered intervention adherence acceptable but not optimal. The difficulty of trying to arrange a weekly group meeting with approximately eight people was evident, although a range of potential times was offered to participants and involved working around the logistics to find the most convenient date. Given the wide variability of participants' availability, simultaneous offers of a group on a weekday early evening or Saturday morning facilitated engagement in groups 2 and 3. In future studies, larger groups will be possible by unblinding investigators after enough participants are allocated to each trial arm to run two groups.

Opting for the group-based and face-to-face design aimed to meet survivors' preferences ${ }^{7}$ but was in contrast with some previous studies reporting proximity as a particular barrier in this population. ${ }^{24}$ The lack of dropouts after the second group session indicated the overall acceptability of the intervention and the favourable rating of most programme aspects provides confidence that only minor content adaptations are needed before testing the study in a large trial. As multiple facilitators will deliver the intervention in a pragmatic setting, future large-scale trials should also measure differences in intervention delivery between various facilitators. Inconvenience and transport were the main barriers to accrual in the current study. Increasing reach might be more feasible with blended designs of group meetings and remote intervention delivery, especially as home-based interventions have typically experienced much lower recruitment rates compared with group-based interventions. In the current programme, even those who adhered mentioned convenience reasons as discouraging participation but the peer support as encouraging. This might suggest delivering some sessions in person and others remotely, potentially through web or mobile technology. A pilot weight loss study with endometrial and breast cancer survivors delivered via a mobile application has shown promising results in a pre-post design. ${ }^{31}$ However, further research on mobile applications for weight management is needed, as most lack evidence-based behaviour change techniques, involvement of healthcare professionals, and scientific evaluation. ${ }^{32}$

In conclusion, this self-help lifestyle intervention trial was feasible in terms of recruitment, adherence and retention. Scaling the trial will require close monitoring of recruitment and attempts should be made to reduce the burden on participants. Further qualitative work could inform a blended in-person and remote design to enhance adherence while retaining the valued peer support. This should be considered before proceeding to a definitive trial. Overall, the lessons learnt from this pilot should inform the design of future studies in this area.

\section{Full protocol availability}

The full protocol of the study has been previously published ${ }^{15}$ and it can also be found in the online supplementary appendix 4 .

\section{Author affiliations}

'Department of Women's Cancer, EGA Institute for Women's Health, University College London, London, UK

${ }^{2}$ Department of Behavioural Science and Health, University College London, London, UK

${ }^{3}$ Leeds Institute of Health Sciences, University of Leeds, Leeds, UK

${ }^{4}$ Department of Gynaecological Oncology, Barts Health NHS Trust, Royal London

Hospital, London, UK

${ }^{5}$ Barts Cancer Institute, Queen Mary University of London, London, UK

${ }^{6}$ Acute Care/Health Systems Division, Yale University School of Nursing, West Haven, Connecticut, USA

Acknowledgements The authors would like to thank the study participants, the second facilitators, the external members of the Trial Steering Committee for the invaluable input in the study and the Institute of Sport Exercise and Health for providing the resources for the study assessments.

Contributors The authors' contributions were as follows. AL and TK conceived the study and were the grant holders. AL and RM were the site investigators for University College London Hospitals and Barts Health, respectively. DAK, AL, RJB and TK initiated the study design and RM helped with protocol development and implementation. DAK and MM recruited the study participants. RJB was responsible for randomisation and auditing. DAK was the trial manager, ran the group sessions, and conducted the baseline and 24-week follow-up assessments. MM conducted the 8-week follow-up assessments. MB provided the statistical support and DAK conducted the statistical analysis. DAK drafted the manuscript, which was amended following comments from all other authors. All authors read and approved the submitted manuscript. All listed authors meet the criteria for authorship and no individual meeting these criteria has been omitted.

Funding This work was supported by the UCL Grand Challenges Scheme, the St. Bartholomew's Hospital Nurses League, the Department of Women's Cancer at The UCL EGA Institute for Women's Health and NIHR University College London Hospitals Biomedical Research Centre, London, UK.

Disclaimer The funders had no role in the study design; collection, management, analysis and interpretation of data; writing of the report; the decision to submit the report for publication.

Competing interests DAK and RJB are volunteers for the charity Weight Concern, which developed the original Shape-Up programme for the general population. All other authors have no conflicts of interest to declare. 
Patient consent Obtained.

Ethics approval The study protocol and documents have been reviewed and approved by the relevant sponsor and National Research Ethics Service Committee London-City Road and Hampstead (Reference: 15/L0/0154).

Provenance and peer review Not commissioned; externally peer reviewed.

Data sharing statement The materials and datasets used and/or analysed during the current study are available from the corresponding author on reasonable request.

Open Access This is an Open Access article distributed in accordance with the Creative Commons Attribution Non Commercial (CC BY-NC 4.0) license, which permits others to distribute, remix, adapt, build upon this work non-commercially, and license their derivative works on different terms, provided the original work is properly cited and the use is non-commercial. See: http://creativecommons.org/ licenses/by-nc/4.0/

(C) Article author(s) (or their employer(s) unless otherwise stated in the text of the article) 2017. All rights reserved. No commercial use is permitted unless otherwise expressly granted.

\section{REFERENCES}

1. Fitzmaurice C, Allen C, Barber RM, et al. Global, Regional, and National Cancer Incidence, Mortality, Years of Life Lost, Years Lived With Disability, and Disability-Adjusted Life-years for 32 Cancer Groups, 1990 to 2015: A Systematic Analysis for the Global Burden of Disease Study. JAMA Oncol 2017;3:524-48.

2. Quaresma M, Coleman MP, Rachet B. 40-year trends in an index of survival for all cancers combined and survival adjusted for age and sex for each cancer in England and Wales, 1971-2011: a populationbased study. Lancet 2015;385:1206-18.

3. Leach CR, Weaver KE, Aziz NM, et al. The complex health profile of long-term cancer survivors: prevalence and predictors of comorbid conditions. J Cancer Surviv 2015;9:239-51.

4. Ward KK, Shah NR, Saenz CC, et al. Cardiovascular disease is the leading cause of death among endometrial cancer patients. Gynecol Oncol 2012;126:176-9.

5. Koutoukidis DA, Knobf MT, Lanceley A. Obesity, diet, physical activity, and health-related quality of life in endometrial cancer survivors. Nutr Rev 2015;73:399-408.

6. von Gruenigen VE, Waggoner SE, Frasure HE, et al. Lifestyle challenges in endometrial cancer survivorship. Obstet Gynecol 2011;117:93-100.

7. Koutoukidis DA, Beeken RJ, Lopes S, et al. Attitudes, challenges and needs about diet and physical activity in endometrial cancer survivors: a qualitative study. Eur J Cancer Care 2016.

8. Zhu G, Zhang X, Wang Y, et al. Effects of exercise intervention in breast cancer survivors: a meta-analysis of 33 randomized controlled trails. Onco Targets Ther 2016;9:2153-68.

9. Stacey FG, James EL, Chapman K, et al. A systematic review and meta-analysis of social cognitive theory-based physical activity and/ or nutrition behavior change interventions for cancer survivors. J Cancer Surviv 2015;9.

10. Morey MC, Snyder DC, Sloane R, et al. Effects of home-based diet and exercise on functional outcomes among older, overweight longterm cancer survivors: RENEW: a randomized controlled trial. JAMA 2009;301:1883-91.

11. von Gruenigen VE, Gibbons HE, Kavanagh MB, et al. A randomized trial of a lifestyle intervention in obese endometrial cancer survivors: quality of life outcomes and mediators of behavior change. Health Qual Life Outcomes 2009;7:17.
12. von Gruenigen V, Frasure H, Kavanagh MB, et al. Survivors of uterine cancer empowered by exercise and healthy diet (SUCCEED): a randomized controlled trial. Gynecol Oncol 2012;125:699-704.

13. Wardle J, Liao LM, Rapoport L, et al. Shape-Up: A lifestyle programme to manage your weight. London: Weight Concern, 2016.

14. Use of intervention mapping to adapt a lifestyle intervention for endometrial cancer survivors International Congress of Behavioral Medicine. Melbourne, Australia, 2016.

15. Koutoukidis DA, Beeken RJ, Manchanda R, et al. Diet and exercise in uterine cancer survivors (DEUS pilot) - piloting a healthy eating and physical activity program: study protocol for a randomized controlled trial. Trials 2016;17:130.

16. Oken MM, Creech $\mathrm{RH}$, Tormey DC, et al. Toxicity and response criteria of the Eastern Cooperative Oncology Group. Am J Clin Oncol 1982;5:649-56.

17. Eldridge SM, Chan CL, Campbell MJ, et al. CONSORT 2010 statement: extension to randomised pilot and feasibility trials. Pilot Feasibility Stud 2016;2:64.

18. Hoffmann TC, Glasziou PP, Boutron I, et al. Better reporting of interventions: template for intervention description and replication (TIDieR) checklist and guide. BMJ 2014;348:g1687.

19. WCRF. Healthy living after cancer. Secondary Healthy living after cancer 2015. https://www.wcrf-uk.org/sites/default/files/healthyliving-after-cancer-guide.pdf

20. Kanarek NF, Kanarek MS, Olatoye D, et al. Removing barriers to participation in clinical trials, a conceptual framework and retrospective chart review study. Trials 2012;13:237.

21. Mills EJ, Seely D, Rachlis B, et al. Barriers to participation in clinical trials of cancer: a meta-analysis and systematic review of patientreported factors. Lancet Oncol 2006;7:141-8.

22. Queensland_Health. Participant Satisfaction Survey. Secondary Participant Satisfaction Survey. 2014 http://www.health.qld.gov.au/ stayonyourfeet/toolkits/phase4/tools-temp.asp

23. Hsieh HF, Shannon SE. Three approaches to qualitative content analysis. Qual Health Res 2005;15:1277-88.

24. Stull VB, Snyder DC, Demark-Wahnefried W. Lifestyle interventions in cancer survivors: designing programs that meet the needs of this vulnerable and growing population. J Nutr 2007;137:243S-8.

25. Denicoff AM, McCaskill-Stevens W, Grubbs SS, et al. The National Cancer Institute-American Society of Clinical Oncology Cancer Trial Accrual Symposium: summary and recommendations. J Oncol Pract 2013;9:267-76.

26. Arem H, Park Y, Pelser $\mathrm{C}$, et al. Prediagnosis body mass index, physical activity, and mortality in endometrial cancer patients. J Natl Cancer Inst 2013;105:342-9.

27. UKDE. Census 2011: Wards in London. Secondary Census 2011: Wards in London 2011. http://ukdataexplorer.com/census/london/\# KS401EW0020

28. Rossi A, Garber CE, Ortiz M, et al. Feasibility of a physical activity intervention for obese, socioculturally diverse endometrial cancer survivors. Gynecol Oncol 2016;142:304-10.

29. Scott E, Daley AJ, Doll H, et al. Effects of an exercise and hypocaloric healthy eating program on biomarkers associated with long-term prognosis after early-stage breast cancer: a randomized controlled trial. Cancer Causes Control 2013;24:181-91.

30. Adams RN, Mosher CE, Blair CK, et al. Cancer survivors' uptake and adherence in diet and exercise intervention trials: an integrative data analysis. Cancer 2015;121.

31. McCarroll ML, Armbruster S, Pohle-Krauza RJ, et al. Feasibility of a lifestyle intervention for overweight/obese endometrial and breast cancer survivors using an interactive mobile application. Gynecol Oncol 2015;137:508-15.

32. Rivera J, McPherson A, Hamilton J, et al. Mobile Apps for Weight Management: A Scoping Review. JMIR Mhealth Uhealth 2016;4:e87. 